\title{
Increased Brain-Derived Neurotrophic Factor Levels in Cerebrospinal Fluid During the Acute Phase in TBI-Induced Mechanical Allodynia in the Rat Model
}

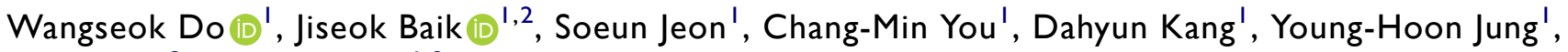 \\ Jiyoon Lee ${ }^{3}$, Hae-Kyu Kim ${ }^{1,2}$ \\ 'Department of Anesthesia and Pain Medicine and Biomedical Research Institute Pusan National University Hospital, Busan, Republic of Korea; \\ ${ }^{2}$ Department of Anesthesia and Pain Medicine, School of Medicine, Pusan National University, Busan, Republic of Korea; ${ }^{3}$ Department of Anesthesia \\ and Pain Medicine, Seoul National University Bundang Hospital, Seongnam-si, Gyeonggi-do, Republic of Korea \\ Correspondence: Jiseok Baik, Department of Anesthesia and Pain Medicine and Biomedical Research Institute Pusan National University Hospital, I79 \\ Gudeok-Ro, Seo-gu, Busan, 4924I, Republic of Korea, Tel +82-5I-240-7499, Fax +82-5I-242-7466, Email jidal75@naver.com
}

Background: The present study aimed to develop a rat model for mechanical allodynia after traumatic brain injury (TBI) and to investigate the expression of brain-derived neurotrophic factor (BDNF) in the cerebrospinal fluid (CSF) using this model.

Methods: A total of 180 rats were randomly allocated into three groups: a control group (group C), a sham-operated group (group S), and a controlled cortical impact induced TBI group (group T), 60 in each group. Von Frey test was performed to evaluate mechanical withdrawal thresholds. An enzyme-linked immunosorbent assay was performed to quantify BDNF level in CSF.

Results: The 50\% withdrawal thresholds of group $\mathrm{T}$ were lower than those of group $\mathrm{C}$ and group $\mathrm{S}$ at all measuring points except for the preoperative period ( $\mathrm{P}=0.026,<0.001$, and $<0.001$ for POD1, POD7, and POD14, respectively). The BDNF level of group T was higher than those of group $\mathrm{C}$ and group $\mathrm{S}$ at POD1 $(\mathrm{P}=0.005)$.

Conclusion: Upregulation of the BDNF expression in CSF was observed in rats who developed mechanical allodynia on the day after TBI. Based on our findings, to elucidate the relationship between TBI-induced neuropathic pain and BDNF expression in CSF, further research should be carried out through a multifaceted approach to a broad spectrum of pain behavior models.

Keywords: brain injuries, traumatic, brain injuries, brain-derived neurotrophic factor, nerve growth factors, neuralgia, models, animal

\section{Introduction}

Traumatic brain injury (TBI) is damage to the brain caused by an external mechanical force. ${ }^{1}$ With industrialization and ever-increasing traffic volumes, the number of TBI has been rising every year. ${ }^{2}$ As medical advances have dramatically improved the survival rate of TBI patients, ${ }^{3}$ the emphasis on prevention, treatment, and rehabilitation for complications following TBI is growing. However, many survivors of TBI suffer from disabling deficits in their cognition and sensorimotor function and from personality changes, and this TBI-associated morbidity not only affects the quality of life of the individual patient but also creates a substantial socioeconomic burden. ${ }^{1}$

Pain is a common problem after TBI, with $32.1-75.3 \%$ of patients with TBI complaining of significant pain. ${ }^{4,5}$ Unlike acute pain, chronic pain following TBI is not directly associated with the extent of the primary tissue damage. ${ }^{4}$ Although the underlying mechanism has not been clearly elucidated, neuropathic pain after TBI seems to develop as a result of the regeneration process of neural tissue after the primary mechanical insult and mainly manifests as allodynia, hyperalgesia, and dysesthesia. Many factors may affect the incidence of neuropathic pain after TBI, and one group that has been actively studied in recent years is neurotrophins, nerve growth factor (NGF), brain-derived neurotrophic factor (BDNF), neurotrophin 3, and neurotrophin $4 .{ }^{6,7}$

Neurotrophins substantially affects the structure and function of the human nervous system. During the developmental period of the embryo, neurotrophins are involved in neurogenesis, neural migration, and synaptogenesis. ${ }^{8,9}$ In adults, 
neurotrophins are associated with synaptic transmission, neuroprotection, neuronal plasticity, and regeneration. ${ }^{8,10,11}$ Given the effects of BDNF on neuronal survival and regeneration, upregulated neurotrophins in CSF after CNS injury ${ }^{12}$ has traditionally been considered a neuroprotective response. ${ }^{10}$ However, recent studies identified that neurotrophins are also involved in peripheral and central sensitization, making them pivotal factors in the pathogenesis of neuropathic pain. ${ }^{7,12}$ In particular, among these neurotrophins, BDNF usually acts on spinal and supraspinal sites ${ }^{7}$ and is expected to be the most important neurotrophin in the pathogenesis of TBI-induced neuropathic pain. However, details of the effect of BDNF on the pathogenesis of neuropathic pain after TBI have not been elucidated to date. ${ }^{6}$

In this context, the present study aimed to develop a rat model for TBI-Induced mechanical allodynia, one of the troublesome components of neuropathic pain, and to investigate the expression of BDNF in the CSF using this model. We also investigated the effect of blood contamination on the concentrations of BDNF in CSF.

\section{Materials and Methods}

\section{Animals}

\section{Experimental Animals, Ethics Statement, and Randomization}

All animal procedures were approved by the Institutional Animal Care and Use Committee at Pusan National University (approval number: PNUH-2019-157). All experiments followed the Guide for the Care and Use of Laboratory Animals published by the United States National Institute of Health (NIH Publication No. 85-23, updated 2011). Six-week-old male Sprague-Dawley rats weighing approximately $200 \mathrm{~g}$ were purchased from Hana Laboratories (Busan, Korea). Rats were housed in a humidity- $(50 \pm 5 \%)$ and temperature-controlled $\left(23 \pm 2{ }^{\circ} \mathrm{C}\right)$ room, under a $12 / 12$ hour light (200 lux)/ dark cycle, and with rodent standard chow and water available ad libitum. Blocked randomization of 180 rats with an internet-based randomizer service (https://www.sealedenvelope.com/simple-randomiser/v1/lists) allocated 60 agematched rats each to either of three groups: a control group (Group C), a sham group (Group S), and a TBI group (Group T). Rats that died during the study were excluded from the analysis.

\section{TBI Modeling}

We used the controlled cortical impact (CCI) method described by Osier et al ${ }^{13}$ to develop a model of mechanical allodynia after TBI in the rats in Group T. After anesthesia with isoflurane ( $5 \mathrm{vol} \%$ for induction, $2.5 \mathrm{vol} \%$ for maintenance with oxygen), the skull of the rat was securely fixed to the stereotactic frame (PinPoint PCI3000 Precision Cortical Impactor; Hatteras Instruments Inc., Cary, NC, USA) in a prone position. After removing the hair with an electric hair clipper, we performed a midline incision of the scalp, abraded the periosteum, and retracted the scalp muscle with an eye speculum to secure sufficient space for the craniotomy. The craniotomy was performed using a mini drill (Saeshin Precision Co., Ltd., Daegu, South Korea) with 5-mm trephine drill bits (Super-Cut Cup Bur $5.0 \mathrm{~mm}$; Maillefer Instruments Holding, Ballaigues, Switzerland) on the midline between the bregma and lambda and $5 \mathrm{~mm}$ to the right of the sagittal suture. The diameter of a cranial window was $5 \mathrm{~mm}$. Considerable care was taken to avoid direct damage to the dura mater during craniotomy. We then removed the bone flap, aligned the tip of the CCI device horizontally with the dura, positioned the tip on the dura, and delivered the impact with a depth of $2.5 \mathrm{~mm}$, velocity of $3 \mathrm{~m} / \mathrm{s}$, and dwell time of $85 \mathrm{~ms}$ (Figure 1). After careful bleeding control, the bone flap was put back, and the wound was closed with a surgical skin stapler (3M $\mathrm{M}^{\mathrm{TM}}$ Precise ${ }^{\mathrm{TM}}$ Vista Disposable Skin Stapler; 3M, St. Paul, MN, USA). After that, the rats were returned to their home cage for recovery. Aseptic techniques were used during all invasive procedures. In the rats of Group S, the same procedures except for the delivery of the CCI were performed. In the rats in Group C, we did not perform any procedure.

\section{Behavior Test}

The von Frey test was performed as described by Kim et al $^{14}$ preoperatively (immediately before surgery), on postoperative day (POD) 1, POD7, and POD14 by a blinded investigator to assess the mechanical threshold in the rats. The measurements on these days were individually performed in a plastic chamber with a mesh screen floor and under dark and quiet conditions at the same time (between 8 and 9 am). All rats had a 15-min habituation period before the test. From underneath the mesh screen, a von Frey filament (BIOSEB, In-Vivo Research Instruments, Vitrolles, France) was perpendicularly applied to the plantar surface of the left hind paw (contralateral to the brain injury) with sufficient force to bend the filament and then held in place for $2 \mathrm{sec}$. The set of von Frey filaments contained the following filaments (number [bending 


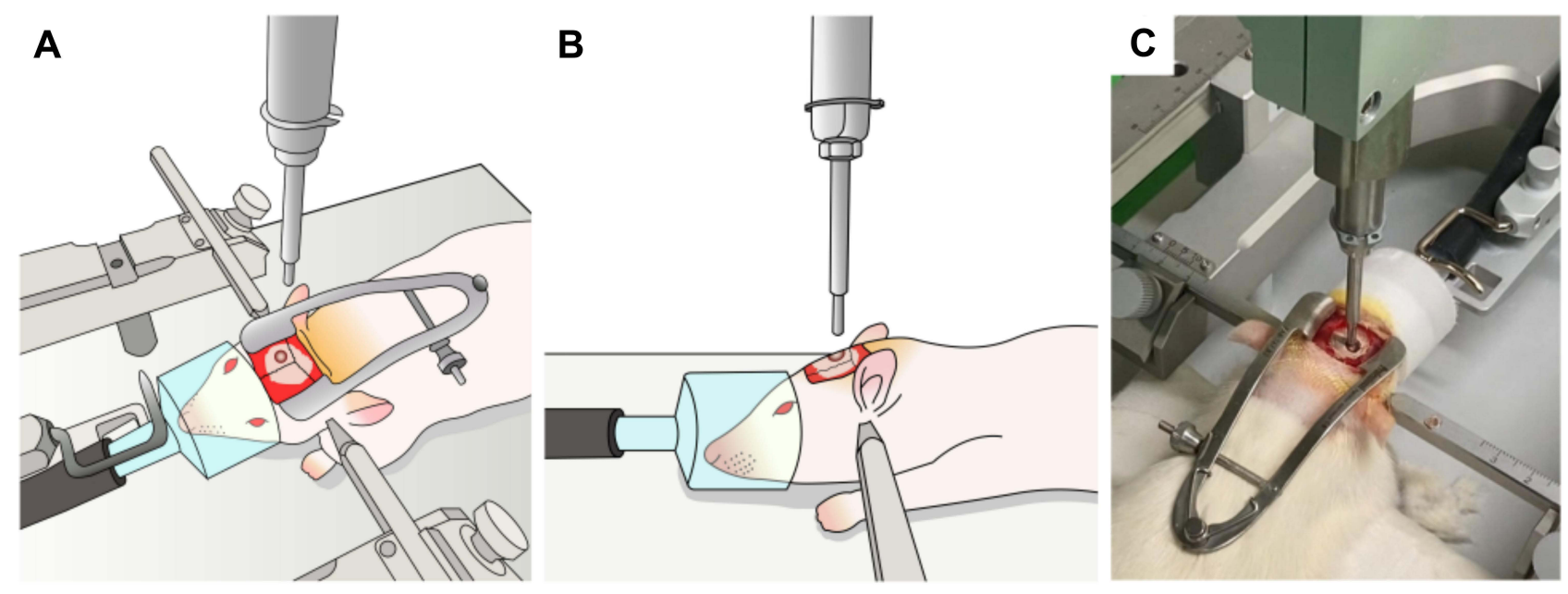

D

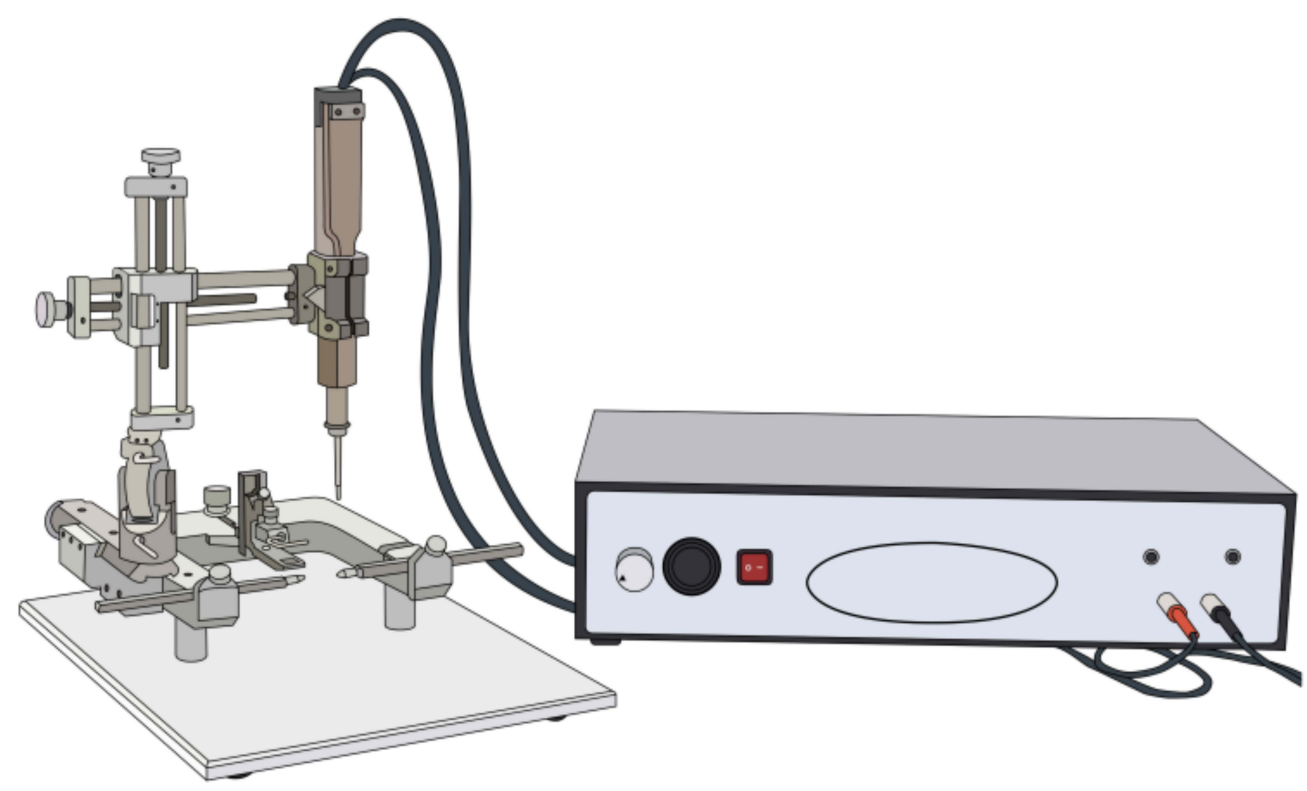

Figure I Delivery of controlled cortical impact in a rat model of traumatic brain injury. We used the controlled cortical impact method to develop a rat model of neuropathic pain after traumatic brain injury. (A-C) After anesthesia with isoflurane, the rat's head was securely fixed to the stereotactic frame in a prone position. A craniotomy was performed, and the impact was delivered (depth $2.5 \mathrm{~mm}$, velocity $3 \mathrm{~m} / \mathrm{s}$, and dwell time $85 \mathrm{~ms}$ ). (D) The controlled cortical impact device with a stereotactic frame (Pinpoint PCI3000 Precision Cortical Impactor; Hatteras Instruments, Cary, NC, USA).

force in g]): 4.31 [2.04], 4.52 [3.31], 4.74 [5.50], 4.92 [8.32], and 5.16 [14.45]. The filaments were applied using the up-down method starting from the weakest filament (4.31). Depending on whether there was a positive or negative response, the next weaker or stronger filament was applied. ${ }^{15}$ A positive response was defined as an abrupt withdrawal or licking of the paw during stimulation or immediately after the removal of the stimulus. The test was continued until six additional stimuli after the first positive response had been recorded. If there were continuously positive or negative responses, the stimulation was considered as a von Frey filament number 3.538 or 5.272 , respectively.

The $50 \%$ threshold value was calculated using Dixon's formula: ${ }^{16} 50 \%$ threshold $=10^{(X+k d)} / 10^{4}$, where $\mathrm{X}$ is the value of the final von Frey filament number in logarithmic units, $\mathrm{k}$ is the tabular value of a positive/negative response, and $d$ is the mean difference between the stimuli in logarithmic units $(0.22)$.

\section{CSF Sampling and BDNF Measurement CSF Sampling}

CSF samples were collected on POD1, POD7, and POD14. After anesthesia with isoflurane (5 vol\% for induction, $2.5 \mathrm{vol} \%$ for maintenance with oxygen), the skull of the rat was securely fixed to the stereotactic frame in a prone position. The axis 
and occipital protuberance of the rat were in line and flexed. The hair was removed with an electric hair clipper, and the puncture site at the atlanto-occipital joint was sterilized with betadine and alcohol. We employed the anatomical landmark approach to identify the exact puncture site: the middle of the triangle demarcated by the occipital protuberance and the most prominent points of the transverse processes of the atlas. A $27 \mathrm{G}$ scale needle $(27 \mathrm{G}$ disposable scalp vein sets, Ningbo Greatcare Co., Ltd., Zhejiang, China) connected bevel-down to a syringe was vertically inserted into the puncture site and advanced to the cisterna magna (Figure 2). Under gentle aspiration, $60-100 \mu \mathrm{L}$ of the CSF flowing into the syringe was collected. The sample was filled into a $1.5 \mathrm{~mL}$ Eppendorf tube and immediately stored in an icebox at $4^{\circ} \mathrm{C}$.

After all procedures (CCI and CSF sampling), rats were closely monitored until 30 min after the emergence of anesthesia. Gait impairment or quadriplegia was assessed 6 hours after the procedure and after that, every 24 hours until sacrifice.

\section{CSF Grading}

The CSF samples were classified into four grades according to the degree of blood contamination (Figure 3): grade 0, colorless and completely transparent; grade 1, pinkish and partially transparent; grade 2, reddish and partially transparent; grade 3, reddish and opaque. As our preliminary results demonstrated that blood contamination could affect the BDNF levels in the CSF, only grade 0 CSF samples were included in the further analysis of BDNF expression in the mechanical
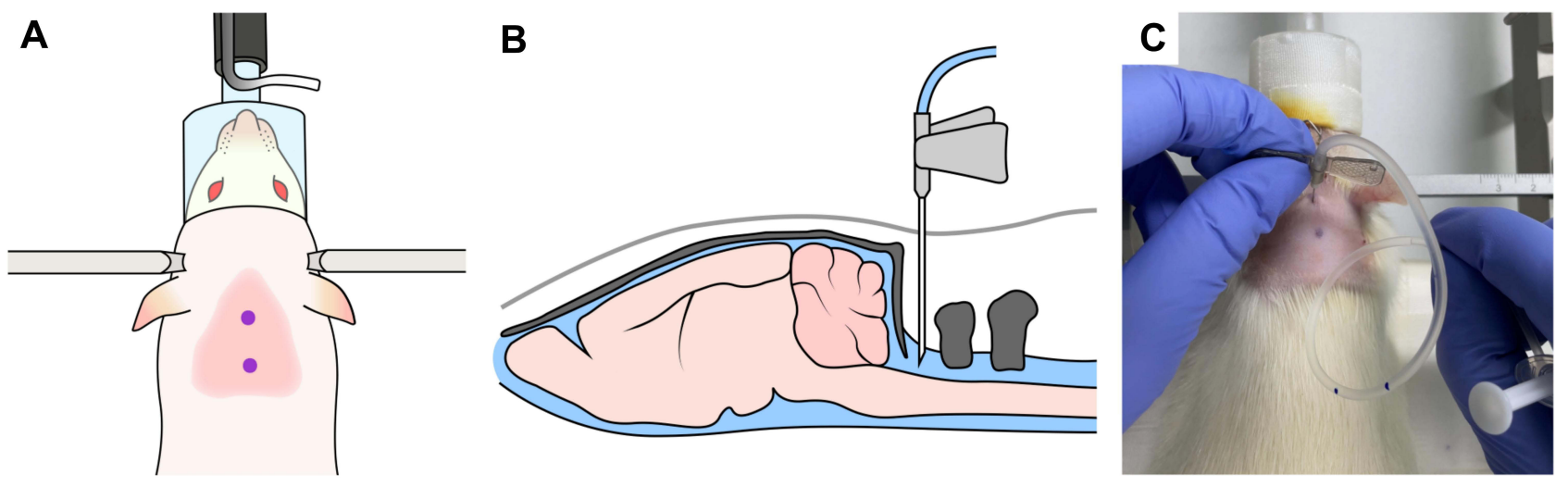

Figure 2 Cerebrospinal fluid sampling in this rat model. (A) An anatomical landmark approach was employed to identify the puncture site above the atlanto-occipital joint in the middle of a triangle between the occipital protuberance and the most prominent points of the transverse processes of the atlas. (B and $\mathbf{C}$ ) A $27 \mathrm{G}$ needle connected to a syringe bevel-down was vertically inserted into the puncture site and advanced to the cisterna magna.
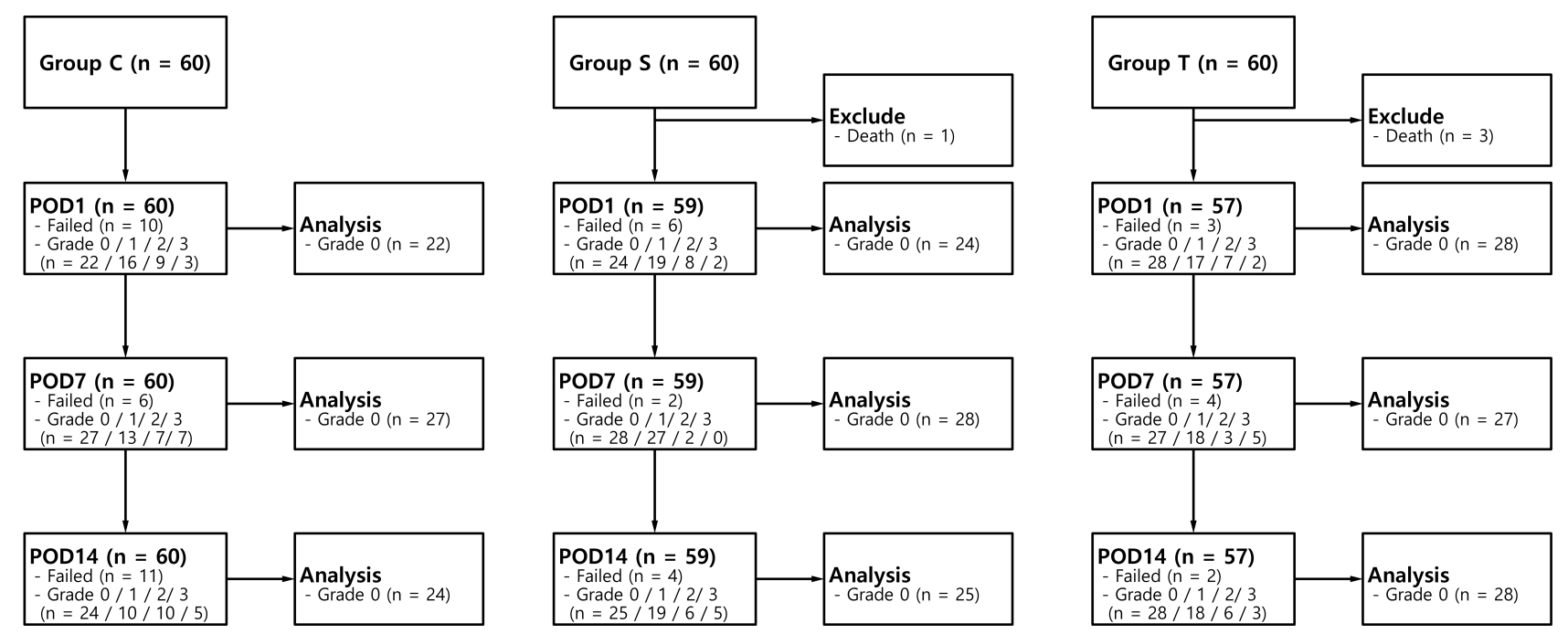

Figure 3 Flow of 180 Sprague-Dawley rats through this study on brain-derived neurotrophic factor levels after traumatic brain injury. Abbreviations: POD, postoperative day; TBI, traumatic brain injury. 
allodynia after the TBI model (Supplements 1 and 2). After the CSF samples were centrifuged at $3000 \mathrm{RPM}$ and $4^{\circ} \mathrm{C}$ for $15 \mathrm{~min}$, the supernatants were collected and stored in a $-80^{\circ} \mathrm{C}$ deep freezer.

\section{Enzyme-Linked Immunosorbent Assay}

The BDNF levels in the CSF were measured using an enzyme-linked immunosorbent assay (RayBio ${ }^{\circledR}$ Rat BDNF ELISA kit; RayBiotech, Norcross, GA, USA) as per the manufacturer's protocol. Briefly, $100 \mu \mathrm{L}$ of standard, blank, and CSF samples were aliquoted in duplicate to a 96-well plate, where the BDNF in the samples was bound to the wells by the immobilized antibody for 150 min. After having been washed with wash buffer, a biotinylated anti-rat BDNF antibody was added, and the plate incubated for $1 \mathrm{hr}$. After washing with wash buffer and horseradish peroxidase-conjugated streptavidin, the wells were incubated for $45 \mathrm{~min}$. The wells were then washed again with buffer, stained with $3,3^{\prime}, 5,5^{\prime}-$ tetramethylbenzidine substrate solution for $30 \mathrm{~min}$ in the dark, and incubated with stop solution. The absorbance at 450 $\mathrm{nm}$ was measured and converted to $\mathrm{pg} / \mathrm{mL}$, the unit for the concentration of BDNF, using a microplate-spectrophotometer (Synergy ${ }^{\mathrm{TM}}$ H1; BioTek Instruments Inc., Winooski, VT, USA). As the samples were loaded in duplicate, each sample's average BDNF value was used as the measurement result.

\section{Subgroup Analysis}

A subgroup analysis was performed on rats in which mechanical allodynia was significantly induced after TBI. The rats excluded for subgroup analysis were as follows: (1) rats in groups $\mathrm{C}$ or $\mathrm{S}$ with a $50 \%$ withdrawal threshold change (decrease or increase higher than 50\%) at POD1 compared to preoperatively, (2) the rats in group $\mathrm{T}$ with a $50 \%$ withdrawal threshold reduction (lower than 50\%) at POD1 compared to preoperatively.

\section{Statistical Analysis}

The primary aim of this study was to investigate the difference in BDNF level in CSF between the three groups (Groups $\mathrm{C}, \mathrm{S}$, and T) in POD1. Due to the lack of previous studies on this subject, it was difficult to predict the appropriate effect size. We determined the sample size based on Cohen's study. ${ }^{17}$ According to Cohen's study, 21 subjects per group were required, with an alpha-value of 0.05 , a beta-value of 0.2 , and a large effect size. In the preliminary experiment, the likelihood of obtaining grade 0 CSF samples was 35\%, and the dropout rate was $65 \%$. Overall, in the present study, 60 subjects per group were required.

All analyses were performed using MedCalc ${ }^{\circledR}$ Statistical Software for Windows, version 18.11.6 (MedCalc Software Ltd., Ostend, Belgium) and IBM SPSS Statistics, version 25 (IBM Corp., Armonk, NY, USA). Categorical data were reported as absolute numbers and percentages and analyzed using the chi-square test. Continuous variables were presented as the mean \pm standard error of the mean (SEM) and analyzed using one-way analysis of variance. If the differences between the groups were significant, the Student-Newman-Keuls tests were performed for posthoc multiple comparisons. The Jonckheere-Terpstra trend test and Spearman correlation analysis were performed between the blood contamination grade and other BDNF concentrations.

A correlation was judged to be weak when the $\mid$ Spearman correlation coefficient (r) $\mid$ was $<0.3$, moderate when $0.3<1$ $\mathrm{r} \mid<0.7$, and strong when $|\mathrm{r}|>0.7$. Two-sided $P$-values $<0.05$ were considered to indicate statistical significance.

\section{Results}

\section{Expression of Neuropathic Pain After TBI in the Rat Model}

Of the 180 rats enrolled, 60,59, and 57 rats were included in the final analysis of group $\mathrm{C}$, group $\mathrm{S}$, and group $\mathrm{T}$, respectively (Figure 3). Four rats were excluded because they died, one in group $\mathrm{S}$ and three in group $\mathrm{T}$. The mean weight in groups $\mathrm{T}$ and $\mathrm{S}$ were statistically significantly lower than that of group $\mathrm{C}$ on POD1 and POD7 $(P<0.001$ and $<0.001$, respectively; Table 1). The $50 \%$ withdrawal thresholds of group $\mathrm{T}$ were lower than those of group $\mathrm{C}$ and group $\mathrm{S}$ at all measuring points except for the preoperative period $(P=0.026,<0.001$, and $<0.001$ for POD1, POD7, and POD14, respectively; Table 2). On POD14, the 50\% withdrawal threshold in group S was also lower than that of group C. 
Table I Weight Change of Each Group Over Time

\begin{tabular}{|c|c|c|c|c|}
\hline & Group C & Group S & Group T & P-value \\
\hline \multicolumn{5}{|l|}{ Whole group } \\
\hline \multicolumn{5}{|l|}{ Weight (g) } \\
\hline Preoperative & $207.1(2.2)$ & $207.3(1.9)$ & $207.9(2.2)$ & 0.957 \\
\hline PODI & $211.0(2.2)^{\dagger}$ & $200.7(1.9)^{*}$ & $197.9(2.3)^{*}$ & $<0.001$ \\
\hline POD7 & $254.2(2.5)^{\dagger}$ & $241.4(1.9)^{*}$ & $241.8(2.3)^{*}$ & $<0.001$ \\
\hline PODI4 & $288.3(2.5)$ & $282.7(1.9)$ & $279.9(3.1)$ & 0.062 \\
\hline \multicolumn{5}{|l|}{ Subgroup } \\
\hline Samples, $\mathbf{n}$ & 31 & 16 & 18 & \\
\hline \multicolumn{5}{|l|}{ Weight (g) } \\
\hline Preoperative & $206.5(3.0)$ & $209.0(3.6)$ & $212.3(3.0)$ & 0.463 \\
\hline PODI & $210.8(2.8)$ & $198.2(3.4)^{*}$ & $201.0(3.1)^{*}$ & 0.010 \\
\hline POD7 & $253.5(3.3)$ & $236.3(2.5)^{*}$ & $245.0(3.6)$ & 0.003 \\
\hline PODI4 & $286.5(3.0)$ & $276.4(3.0)$ & 286.0 & 0.162 \\
\hline
\end{tabular}

Notes: Variables were presented as the mean \pm SEM. Group $C$, control; Group $S$, sham surgery; group T, traumatic brain injury; $* P<0.05$ compared to the control. ${ }^{\dagger} P<0.05$ compared to sham. Bold text; statistically significant data.

Abbreviations: POD, postoperative day; SEM, standard error of the mean.

Table 2 50\% Withdrawal Threshold Change of Each Group Over Time

\begin{tabular}{|c|c|c|c|c|}
\hline & Group C & Group S & Group $\mathbf{T}$ & $P$-value \\
\hline \multicolumn{5}{|l|}{ Whole group } \\
\hline Samples, $n$ & 60 & 59 & 57 & \\
\hline \multicolumn{5}{|l|}{$50 \%$ withdrawal threshold (g) } \\
\hline Preoperative & $13.8(0.6)$ & $13.6(0.6)$ & $13.3(0.7)$ & 0.862 \\
\hline PODI & I $4.3(0.6)$ & I4.I(0.7) & I I.7 (0.9)*t & 0.026 \\
\hline POD7 & $13.7(0.7)$ & I3.| (0.8) & $9.6(0.8)^{* t}$ & $<0.001$ \\
\hline PODI4 & $15.2(0.6)^{\dagger}$ & $12.7(0.7)^{*}$ & $10.5(0.8)^{* t}$ & $<0.001$ \\
\hline \multicolumn{5}{|l|}{ Subgroup } \\
\hline Samples, $\mathbf{n}$ & 31 & 16 & 18 & \\
\hline \multicolumn{5}{|l|}{$50 \%$ withdrawal threshold (g) } \\
\hline Preoperative & $14.9(0.7)$ & $15.8(1.3)$ & $17.6(0.8)$ & 0.083 \\
\hline PODI & $14.8(0.3)$ & $15.9(1.1)$ & $6.0(0.4)^{* \dagger}$ & $<0.001$ \\
\hline POD7 & $14.7(0.9)^{\dagger}$ & II.I (1.6)* & $7.6(0.6) *$ & $<0.001$ \\
\hline PODI4 & $15.5(0.7)^{\dagger}$ & $9.2(1.1)^{*}$ & $8.5(1.3)^{*}$ & $<0.001$ \\
\hline
\end{tabular}

Notes: Variables were presented as the mean \pm SEM. Group C, control; Group S, sham surgery; group T, traumatic brain injury; ${ }^{* P}<0.05$ compared to the control. ${ }^{\dagger} P<0.05$ compared to sham. Bold text; statistically significant data.

Abbreviations: POD, postoperative day; SEM, standard error of the mean.

\section{BDNF Expression in the CSF}

The CSF samples included in this analysis are shown in Figure 3. The BDNF level of group $\mathrm{T}$ was statistically significantly higher than those of group $\mathrm{C}$ and group $\mathrm{S}$ at POD1 $(P=0.005$; Table 3$)$.

\section{Subgroup Analysis}

For subgroup analysis, 31, 16, and 18 rats were included in group C, group S, and group T, respectively (Figure 4). The tendency was also maintained in the subgroup analysis. The mean weight in groups $\mathrm{T}$ and $\mathrm{S}$ were lower than that of group $\mathrm{C}$ on POD1 $(P=0.010$; Table 1); and the mean weight in groups T was lower than that of group $\mathrm{C}$ on POD7 $(P=0.003$; 
Table 3 Brain-Derived Neurotrophic Factor in Cerebrospinal Fluid Samples without Blood (Grade 0)

\begin{tabular}{|c|c|c|c|c|}
\hline \multirow{2}{*}{} & \multicolumn{3}{|c|}{ BDNF (pg/mL), (Mean, SEM) [n] } & \multirow{2}{*}{ P-value } \\
\cline { 2 - 5 } & Group C & Group S & Group T & \\
\hline Whole group & $9.3(1.2)[22]$ & $11.1(0.5)[24]$ & $16.8(2.2)[28]^{* \dagger}$ & 0.005 \\
\hline POD I & $10.2(1.4)[27]$ & $10.8(1.4)[28]$ & $13.4(0.9)[27]$ & 0.368 \\
POD 7 & $6.2(1.1)[24]$ & $10.0(2.3)[25]$ & $9.6(1.6)[28]$ & 0.252 \\
POD 14 & \multicolumn{5}{|c|}{} \\
\hline Subgroup & $7.1(1.3)[11]$ & $7.7(1.5)[8]$ & $16.3(6.1)[4]^{*} \dagger$ & 0.050 \\
\hline POD I & $9.3(1.5)[14]$ & $11.2(0.1)[11]$ & $11.7(2.5)[5]$ & 0.638 \\
POD 7 & $4.5(0.7)[12]$ & $11.7(0.1)[9]$ & $4.7(0.2)[4]$ & 0.2877 \\
POD 14 &
\end{tabular}

Notes: ${ }^{*} p<0.05$ as compared to the control. ${ }^{\dagger} p<0.05$ compared to sham. Bold text; statistically significant data.

Abbreviations: BDNF, brain-derived neurotrophic factor; SEM, standard error of the mean; POD, postoperative day; TBI, traumatic brain injury; NGF, nerve growth factor; BDNF, brain-derived neurotrophic factor; CSF, cerebrospinal fluid; Group C, a control group; Group S, a sham group; Group T, a TBI group; $\mathrm{CCl}$, controlled cortical impact; POD, postoperative day; SEM, standard error of the mean; r, Spearman correlation coefficient.

Table 1). The $50 \%$ withdrawal threshold of group T were lower than those of group $\mathrm{C}$ and group $\mathrm{S}$ on POD1 $(P<0.001$; Table 2). The $50 \%$ withdrawal threshold of group $\mathrm{T}$ and $\mathrm{S}$ were lower than those of group $\mathrm{C}$ on POD7 and POD14 $(P<0.001$ and $<0.001$, respectively; Table 2$)$. The BDNF level of group $\mathrm{T}$ was higher than those of group $\mathrm{C}$ and $\mathrm{S}$ at POD $1(P=0.050$; Table 3).

\section{Discussion}

BDNF is the most abundant and universally distributed neurotrophin in the CNS. ${ }^{18,19}$ BDNF plays an essential role in various neural processes throughout the entire human life cycle. ${ }^{8}$ Recent studies have identified BDNF as a new therapeutic target in various neurological and psychiatric disorders. ${ }^{20}$ Systematic reviews and meta-analyses have provided further evidence supporting this approach by showing that blood BDNF levels are lower in patients with Alzheimer's disease $\left(-0.282\left[-0.535^{--0.028]}\right),{ }^{21}\right.$ Parkinson's disease $(-1.03[-1.83-0.23]),{ }^{22}$ depression $(-0.71[-0.89--0.53]),{ }^{23}$ and

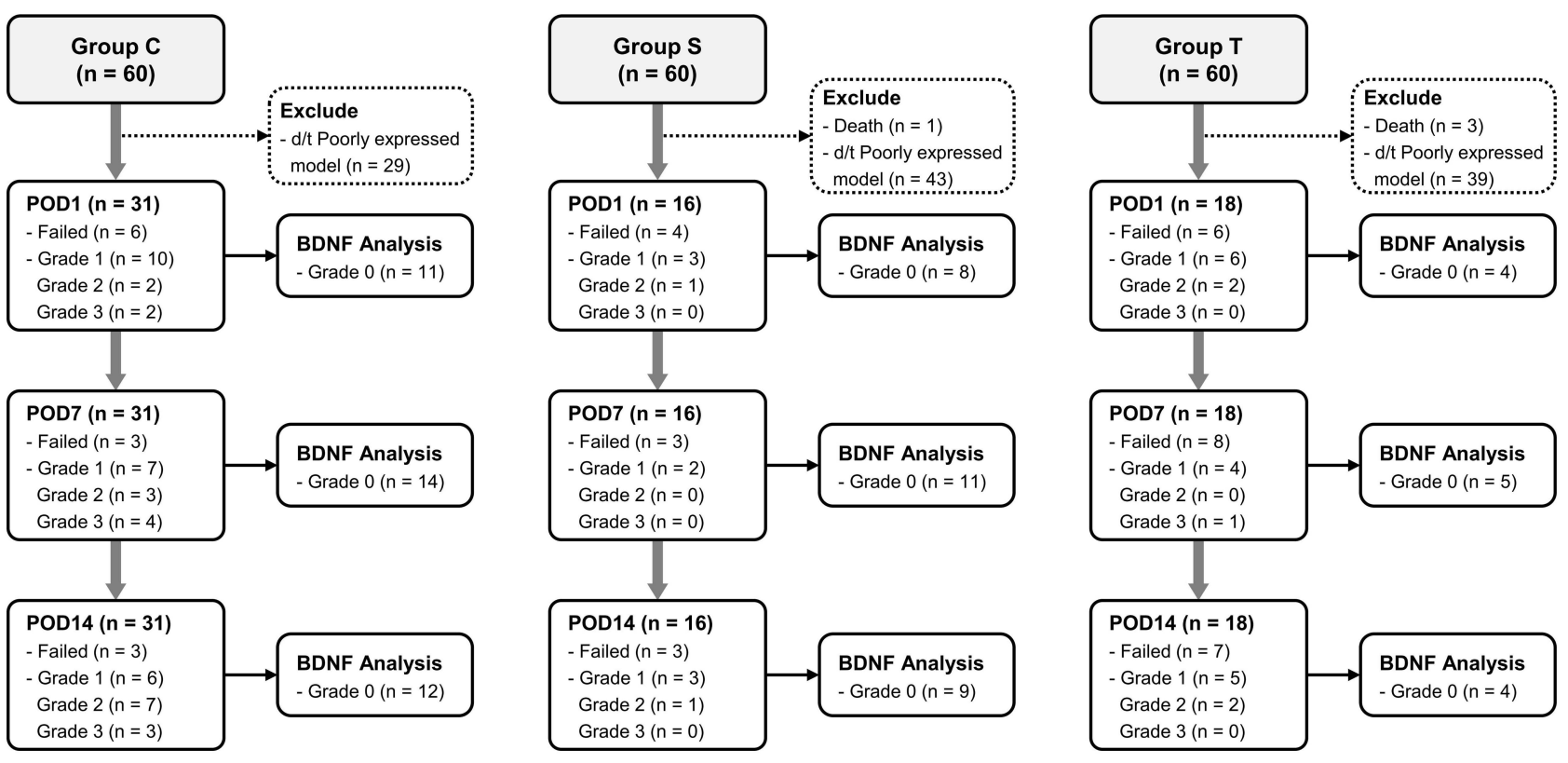

Figure 4 Flow chart of subgroup analysis.

Abbreviations: POD, postoperative day; TBI, traumatic brain injury. 
schizophrenia $(-0.74[-0.99--0.50])^{24}$ but higher in patients with autism $(0.47[0.07-0.86])^{25}$ and post-traumatic stress disorder $(0.52[0.18-0.85])^{19}$ as compared to healthy controls (standardized mean differences [95\% confidence interval]).

After TBI, the increase in CSF BDNF level is thought to be due to the following mechanism. First, after TBI, microglia in the CNS undergo activation and produce BDNF. ${ }^{26,27} \mathrm{In} \mathrm{rat}^{27}$ and primate ${ }^{26}$ models, there is evidence showing microglia as a source of BDNF after brain injury. Second, blood-brain barrier (BBB) disruption occurs after TBI; platelets dump BDNF, and serum BDNF transit into the CNS. Failla et $\mathrm{al}^{12}$ reported that CSF and serum BDNF levels tended to be positively correlated in healthy controls, but that CSF and serum BDNF levels were negatively correlated in TBI patients. The authors also demonstrated that elevated BDNF levels in the CSF were associated with increased mortality and poor prognosis. BDNF also contributes to the development of neuropathic pain due to its implication in peripheral and central sensitization. ${ }^{7}$ However, relevant research on the role of BDNF in neuropathic pain following TBI is scarce. ${ }^{6}$

We developed a model for mechanical allodynia after TBI in rats using the CCI method. ${ }^{13}$ Mechanical allodynia and BDNF levels in the CSF were measured to validate the successful establishment of our animal model. In this study, the $50 \%$ withdrawal thresholds of Group C were 13.8 and $15.2 \mathrm{~g}$ at preoperative and POD 14, showing an increasing trend with time, which is presumed to be the effect of age-related change. Ririe demonstrated that the mechanical withdrawal thresholds of 2, 4, and 16-week-old naïve rats were 5.9 (0.4), 19 (0.8), and 29 (2.5) g (mean [SEM]), respectively. ${ }^{28}$ In addition, in Ririe's subsequent study, the time-dependent increase in the mechanical withdrawal threshold was also reproduced. ${ }^{29}$ The previous results emphasize the need to consider age as a covariate in studies using the rat model of mechanical allodynia. To adjust for the age as a covariate, we designed the present study to evaluate changes in the $50 \%$ withdrawal threshold as compared to naive control of the same age, rather than as within-group comparisons over time. According to our results, the rats in Group $\mathrm{T}$ demonstrated that the $50 \%$ withdrawal threshold of the contralateral paw significantly decreased compared with that of the control group after the operation, suggesting that a rat model for neuropathic pain after TBI was successfully developed. Furthermore, BDNF levels were higher in the TBI model in the acute phase compared to those in groups S and C. Consequently, we conclude that an increase in the acute phase of BDNF level in CSF has an effect on the occurrence of neuropathic pain after TBI.

Chronic pain has a higher prevalence in patients with mild TBI than in patients with moderate to severe TBI. ${ }^{5}$ It is presumed that, neurological deficits such as motor dysfunction, language deficits, and memory disturbance frequently accompany moderate to severe TBI, and the accompanying pain is highly likely to be masked without recognition. ${ }^{5}$ To evaluate neuropathic pain using the von Frey filament test, we designed the study to induce mild TBI that preserves gross motor function. We determined the set point of CCI based on Schonfeld et al who evaluated motor deficits in a rat model of CCI, an impact depth of $5 \mathrm{~mm}$ and a velocity of $3 \mathrm{~m} / \mathrm{sec}^{30}$ The authors also reported that, after CCI-induced TBI, rats showed impaired fine motor skills, but did not demonstrate impaired gross motor skills as evaluated using a gait test, nor did they have quadriplegia. In the present study, the CCI intensity was set at impact depth of $2.5 \mathrm{~mm}$ and velocity of $3 \mathrm{~m} / \mathrm{s}$, which was weaker than that of Schonfeld's model, ${ }^{30}$ and no rats with gait disturbance or quadriplegia after CCI were observed.

Macolino et $\mathrm{al}^{31}$ reported that periorbital and forepaw mechanical allodynia, but not hind paw allodynia, occurred after CCI-induced TBI in rats. In a study using a rat lateral fluid percussion TBI model, mechanical allodynia was induced on the contralateral side compared to a sham operation, and the sensitization persisted up to three days. ${ }^{32}$ The authors also reported that BDNF levels were upregulated in the contralateral lumbar spinal cord segment for one week after TBI. These results are generally consistent with our findings. However, our model of neuropathic pain after TBI demonstrated mechanical allodynia that lasted for more than two weeks in the contralateral hind paw. These inconsistent findings may be attributed to the differences in the modeling method (fluid percussion injury vs CCI) and the severity of TBI. Therefore, further well-designed research is needed to establish a predictable, reproducible, and standardized rat model for neuropathic pain after TBI.

It is well documented that a sham operation, ie, craniotomy itself, may induce significant brain damage and cause neuroinflammatory, structural, and behavioral change. ${ }^{33}$ Our study also demonstrated that rats developed mechanical allodynia after the sham operation. On POD14, the 50\% withdrawal threshold in group S was lower than that of group 
$\mathrm{C}$ but higher than that of group T. On POD1, the BDNF levels were higher in group $\mathrm{T}$ than in group S, and the latter were higher than those in group $\mathrm{C}$.

In this study, instead of harvesting CNS tissue, we used CSF sampling to measure serial BDNF concentrations. Iatrogenic blood contamination of the CSF is very common ${ }^{34}$ and was observed in $55.9 \%$ of the CSF samples in our study. For a quick assessment without additional equipment, we used visual inspection to classify the amount of blood contamination of the CSF. Ylitalo et $\mathrm{al}^{34}$ reported that when CSF samples of rats are slightly reddish or clearly red on visual inspection, the erythrocyte count may be estimated to be about $2000 \times 10^{3} / \mathrm{mL}$ or $10,000 \times 10^{3} / \mathrm{mL}$, respectively.

Furthermore, our preliminary research show that the higher the blood contamination, the higher the BDNF concentration of the CSF. This pattern may result because BDNF is also synthesized in vascular endothelial cells of the brain and distributed into the blood. ${ }^{20}$ Blood contamination also affects the level of other biomarkers in the CSF, such as total protein, albumin, and immunoglobulin concentration. ${ }^{34,35}$ To date, there is no consensus on the acceptable blood contamination when measuring BDNF in CSF. Based on our research results, blood contamination should be minimized for the correct measurement of BDNF levels. If blood contamination occurs in the sample, it can be said that it is not suitable as a sample for correct measurement. In addition, previous studies that performed CSF sampling for measuring BDNF considered it challenging to estimate the degree of blood contamination and how much it affected the level of BDNF. ${ }^{12}$ Therefore, further research on CSF sampling is required to develop a reliable adjustment method for blood contamination.

It is known that BDNF evokes pronociceptive signals and plays a central role during the peripheral and central sensitization in inflammatory and neuropathic pain conditions. ${ }^{7}$ Our findings suggest that upregulation of the BDNF expression in CSF is associated with the development of mechanical allodynia during the early acute phase of TBI. However, it remains unclear how long the increase in BDNF in the acute phase after TBI affects the development of mechanical allodynia. Considering the results of the previous study using another animal model, ${ }^{35}$ it can be presumed that acute short-term BDNF increase followed by TBI could induce mechanical allodynia that persists for a considerable time. In a rat cystitis model, Ding et al previously demonstrated that intrathecal injection of exogenous BDNF exacerbated mechanical allodynia, lasting more than ten days. ${ }^{35}$ Further work-up should be performed to determine how long the acute short-term BDNF increase affects neuropathic pain following TBI and to find potential candidate drugs that can inhibit the upregulation of BDNF during this early phase.

The present study has several limitations. First, this study was performed on a rat model of mechanical allodynia after TBI. Therefore, care should be taken when extrapolating our findings to humans. Second, among the various associated factors of mechanical allodynia after TBI, we evaluated only BDNF. In order to elucidate the pathophysiologic mechanisms of mechanical allodynia after TBI, further factors should be studied, particularly other neurotrophins, such as NCF, neurotrophin 3, and neurotrophin 4. Third, the extent of TBI-induced neuronal cell apoptosis was not considered in our study. In this respect, Whiteside et al demonstrated that neuronal cell apoptosis contributes to the initiation and maintenance of neuropathic pain. ${ }^{36}$ In the present study, the CCI intensity of the TBI-induced neuropathic pain model was all set to a single value (impact depth: $2.5 \mathrm{~mm}$, velocity: $3 \mathrm{~m} / \mathrm{s}$ ) to induce mild TBI that preserves gross motor function. Therefore, it is presumed that the extent of neuronal cell apoptosis does not significantly differ withingroup T. Further well-designed CCI intensity-response study is needed to assess the pathophysiology of TBI-induced mechanical allodynia better. Fourthly, in the present study, all subjects who received TBI were included as group T, rather than selectively including only subjects who developed neuropathic pain after TBI. The reason is that it is practically difficult to identify clearly the development of neuropathic pain after TBI. To date, there was no standardized definition for neuropathic pain in the rat model of TBI-induced neuropathic pain. Finally, our study focused only on mechanical allodynia after TBI. Neuropathic pain is defined as pain caused by a lesion or disease of the somatosensory system, and the somatosensory system allows for the perception of touch, pressure, pain, temperature, position, movement, and vibration. ${ }^{37}$ Although mechanical allodynia is the most common symptom of neuropathic pain, ${ }^{38}$ this study is insufficient to represent neuropathic pain that comprises a wide range of heterogeneous conditions. ${ }^{39}$ To date, there is no single behavioral assay capable of capturing the full spectrum of nociception in animal subjects. ${ }^{40}$ To elucidate the various aspects of TBI-induced neuropathic pain, follow-up studies with multifaceted approaches to pain 
behaviors other than mechanical stimuli (eg, thermal, electrical, or chemical stimuli-evoked, or non-stimulatory nociception) are needed.

\section{Conclusion}

In conclusion, upregulation of the BDNF expression in CSF was observed in rats who developed mechanical allodynia on the day after TBI. Based on our findings, to elucidate the relationship between TBI-induced neuropathic pain and BDNF expression in CSF, further research should be carried out through a multifaceted approach to a broad spectrum of pain behavior models.

\section{Ethics Approval}

All animal procedures were approved by the Institutional Animal Care and Use Committee at Pusan National University (approval number: PNUH-2019-157). All experiments followed the Guide for the Care and Use of Laboratory Animals published by the United States National Institute of Health (NIH Publication No. 85-23, updated 2011).

\section{Funding}

This work was supported by the National Research Foundation of Korea (NRF) grant funded by the Korea government (Ministry of Science and ICT) (No. 2018R1C1B6 004841).

\section{Disclosure}

Prof. Dr. Wangseok Do reports grants from National Research Foundation of Korea (NRF) grant funded by the Korean government (Ministry of Science and ICT), during the conduct of the study. Dr Soeun Jeon reports grants from National Research Foundation of Korea (NRF) grant funded by the Korean government (Ministry of Science and ICT), during the conduct of the study. Dr Chang-Min You reports grants from National Research Foundation of Korea (NRF) grant funded by the Korean government (Ministry of Science and ICT), during the conduct of the study. Dr Dahyun Kang reports grants from National Research Foundation of Korea (NRF) grant funded by the Korean government (Ministry of Science and ICT), during the conduct of the study. Dr Jiyoon Lee reports grants from Pusan National University Hospital, grants from Korean government, during the conduct of the study. The authors report no conflicts of interest in this work.

\section{References}

1. Xiong Y, Mahmood A, Chopp M. Animal models of traumatic brain injury. Nat Rev Neurosci. 2013;14(2):128-142. doi:10.1038/nrn3407

2. Jung HY, Han SH. Depression after traumatic brain injury. Korean J Biol Psychiatry. 1999;6(1):21-29.

3. Harrison-Felix CL, Whiteneck GG, Jha A, DeVivo MJ, Hammond FM, Hart DM. Mortality over four decades after traumatic brain injury rehabilitation: a Retrospective Cohort Study. Arch Phys Med Rehabil. 2009;90(9):1506-1513. doi:10.1016/j.apmr.2009.03.015

4. Irvine KA, Clark DJ. Chronic pain after traumatic brain injury: pathophysiology and pain mechanisms. Pain Med. 2018;19(7):1315-1333. doi: $10.1093 / \mathrm{pm} / \mathrm{pnx} 153$

5. Nampiaparampil DE. Prevalence of chronic pain after traumatic brain injury: a systematic review. JAMA. 2008;300(6):711-719. doi:10.1001/ jama.300.6.711

6. Baik J. Neurotrophins and neuropathic pain in patients with traumatic brain injury. Korean J Pain. 2020;33(1):1-2. doi:10.3344/kjp.2020.33.1.1

7. Khan N, Smith MT. Neurotrophins and neuropathic pain: role in pathobiology. Molecules. 2015;20(6):10657-10688. doi:10.3390/ molecules200610657

8. Grande I, Fries GR, Kunz M, Kapczinski F. The role of BDNF as a mediator of neuroplasticity in bipolar disorder. Psychiatry Investig. 2010;7 (4):243-250. doi:10.4306/pi.2010.7.4.243

9. Ghassabian A, Sundaram R, Chahal N, et al. Determinants of neonatal brain-derived neurotrophic factor and association with child development. Dev Psychopathol. 2017;29(4):1499-1511. doi:10.1017/S0954579417000414

10. Song XY, Li F, Zhang FH, Zhong JH, Zhou XF. Peripherally-derived BDNF promotes regeneration of ascending sensory neurons after spinal cord injury. PLoS One. 2008;3(3):e1707. doi:10.1371/journal.pone.0001707

11. Rauti R, Cellot G, D'Andrea P, et al. BDNF impact on synaptic dynamics: extra or intracellular long-term release differently regulates cultured hippocampal synapses. Mol Brain. 2020;13(1):43. doi:10.1186/s13041-020-00582-9

12. Failla MD, Conley YP, Wagner AK. Brain-Derived Neurotrophic Factor (BDNF) in traumatic brain injury-related mortality: interrelationships between genetics and acute systemic and central nervous system BDNF profiles. Neurorehabil Neural Repair. 2016;30(1):83-93. doi:10.1177/ 1545968315586465

13. Osier ND, Korpon JR, Dixon CE. Controlled cortical impact model. In: Kobeissy FH, editor. Brain Neurotrauma: Molecular, Neuropsychological, and Rehabilitation Aspects. 1st ed. Boca Raton (FL): CRC Press/Taylor \& Francis; 2015:177-189. 
14. Kim HY, Lee I, Chun SW, Kim HK. Reactive oxygen species donors increase the responsiveness of dorsal horn neurons and induce mechanical hyperalgesia in rats. Neural Plast. 2015;2015:1-10.

15. Chaplan SR, Bach FW, Pogrel JW, Chung JM, Yaksh TL. Quantitative assessment of tactile allodynia in the rat paw. J Neurosci Methods. 1994;53 (1):55-63. doi:10.1016/0165-0270(94)90144-9

16. Dixon WJ. Efficient analysis of experimental observations. Annu Rev Pharmacol Toxicol. 1980;20(1):441-462. doi:10.1146/annurev. pa.20.040180.002301

17. Cohen J. A power primer. Psychol Bull. 1992;112(1):155-159. doi:10.1037/0033-2909.112.1.155

18. Balaratnasingam S, Janca A. Brain derived neurotrophic factor: a novel neurotrophin involved in psychiatric and neurological disorders. Pharmacol Ther. 2012;134(1):116-124. doi:10.1016/j.pharmthera.2012.01.006

19. Mojtabavi H, Saghazadeh A, van den Heuvel L, Bucker J, Rezaei N. Peripheral blood levels of brain-derived neurotrophic factor in patients with posttraumatic stress disorder (PTSD): a systematic review and meta-analysis. PLoS One. 2020;15(11):e0241928. doi:10.1371/journal.pone.0241928

20. Marie C, Pedard M, Quirié A, et al. Brain-derived neurotrophic factor secreted by the cerebral endothelium: a new actor of brain function? J Cereb Blood Flow Metab. 2018;38(6):935-949. doi:10.1177/0271678X18766772

21. Ng TKS, Ho CSH, Tam WWS, Kua EH, Ho RCM. Decreased serum brain-derived neurotrophic factor (BDNF) levels in patients with Alzheimer's disease (AD): a systematic review and meta-analysis. Int J Mol Sci. 2019;20(2):257. doi:10.3390/ijms20020257

22. Jiang L, Zhang H, Wang C, Ming F, Shi X, Yang M. Serum level of brain-derived neurotrophic factor in Parkinson's disease: a meta-analysis. Prog Neuropsychopharmacol Biol Psychiatry. 2019;88:168-174. doi:10.1016/j.pnpbp.2018.07.010

23. Molendijk ML, Spinhoven P, Polak M, Bus BAA, Penninx BWJH, Elzinga BM. Serum BDNF concentrations as peripheral manifestations of depression: evidence from a systematic review and meta-analyses on 179 associations (N=9484). Mol Psychiatry. 2014;19(7):791-800. doi:10.1038/mp.2013.105

24. Cui H, Jin Y, Wang J, Weng X, Li C. Serum brain-derived neurotrophic factor (BDNF) levels in schizophrenia: a systematic review. Shanghai Arch Psychiatry. 2012;24(5):250-261. doi:10.3969/j.issn.1002-0829.2012.05.002

25. Saghazadeh A, Rezaei N. Brain-derived neurotrophic factor levels in autism: a systematic review and meta-analysis. J Autism Dev Disord. 2017;47 (4):1018-1029. doi:10.1007/s10803-016-3024-x

26. Nagamoto-Combs K, McNeal DW, Morecraft RJ, Combs CK. Prolonged microgliosis in the rhesus monkey central nervous system after traumatic brain injury. J Neurotrauma. 2007;24(11):1719-1742. doi:10.1089/neu.2007.0377

27. Madinier A, Bertrand N, Mossiat C, et al. Microglial involvement in neuroplastic changes following focal brain ischemia in rats. PLoS One. 2009; 4 (12):e8101. doi:10.1371/journal.pone.0008101

28. Ririe DG, Vernon TL, Tobin JR, Eisenach JC. Age-dependent responses to thermal hyperalgesia and mechanical allodynia in a rat model of acute postoperative pain. Anesthesiology. 2003;99(2):443-448. doi:10.1097/00000542-200308000-00027

29. Ririe DG, Eisenach JC. Age-dependent responses to nerve injury-induced mechanical allodynia. Anesthesiology. 2006;104(2):344-350. doi:10.1097/00000542-200602000-00021

30. Schönfeld LM, Jahanshahi A, Lemmens E, et al. Long-term motor deficits after controlled cortical impact in rats can be detected by fine motor skill tests but not by automated gait analysis. J Neurotrauma. 2017;34(2):505-516. doi:10.1089/neu.2016.4440

31. Macolino CM, Daiutolo BV, Albertson BK, Elliott MB. Mechanical allodynia induced by traumatic brain injury is independent of restraint stress. J Neurosci Methods. 2014;226:139-146. doi:10.1016/j.jneumeth.2014.01.008

32. Feliciano DP, Sahbaie P, Shi X, Klukinov M, Clark JD, Yeomans DC. Nociceptive sensitization and BDNF up-regulation in a rat model of traumatic brain injury. Neurosci Lett. 2014;583:55-59. doi:10.1016/j.neulet.2014.09.030

33. Cole JT, Yarnell A, Kean WS, et al. Craniotomy: true sham for traumatic brain injury, or a sham of a sham? J Neurotrauma. 2011;28(3):359-369. doi:10.1089/neu.2010.1427

34. Ylitalo P, Heikkinen ER, Myllylä VV. Evaluation of successive collections of cisternal cerebrospinal fluid in rats, rabbits, and cats. Exp Neurol. 1976;50(2):330-336. doi:10.1016/0014-4886(76)90008-X

35. Ding H, Chen J, Su M, et al. BDNF promotes activation of astrocytes and microglia contributing to neuroinflammation and mechanical allodynia in cyclophosphamide-induced cystitis. J Neuroinflammation. 2020;17(1):19. doi:10.1186/s12974-020-1704-0

36. Whiteside GT, Munglani R. Cell death in the superficial dorsal horn in a model of neuropathic pain. J Neurosci Res. 2001;64(2):168-173. doi:10.1002/jnr.1062

37. Colloca L, Ludman T, Bouhassira D, et al. Neuropathic pain. Nat Rev Dis Prim. 2017;3:17002. doi:10.1038/nrdp.2017.2

38. Yu Y, Wei G, Zhou Q, et al. ErbB4 in spinal PV interneurons regulates mechanical allodynia in neuropathic pain via modulation of glycinergic inhibitory tone. J Pain Res. 2021;14:1643-1651. doi:10.2147/JPR.S311894

39. Fornasari D. Pharmacotherapy for neuropathic pain: a review. Pain Ther. 2017;6(S1):25-33. doi:10.1007/s40122-017-0091-4

40. Deuis JR, Dvorakova LS, Vetter I. Methods used to evaluate pain behaviors in rodents. Front Mol Neurosci. 2017;10:284. doi:10.3389/ fnmol.2017.00284

Journal of Pain Research

Dovepress

Publish your work in this journal

The Journal of Pain Research is an international, peer reviewed, open access, online journal that welcomes laboratory and clinical findings in the fields of pain research and the prevention and management of pain. Original research, reviews, symposium reports, hypothesis formation and commentaries are all considered for publication. The manuscript management system is completely online and includes a very quick and fair peer-review system, which is all easy to use. Visit http://www.dovepress.com/testimonials.php to read real quotes from published authors.

Submit your manuscript here: https://www.dovepress.com/journal-of-pain-research-journal 